\title{
LA SURFACE LIBRE ET LES CONDITIONS DE SIMILITUDE DU VORTEX
}

\section{par G. HOLTORFF *}

\section{Introduction.}

Par comparaison des énergies mises en jeu, on peut démontrer que, lorsque la hauteur de liquide au-dessus de l'orifice de sortie est faible, l'écoulement n'est alors seulement possible que s'il est superposé à un écoulement rotatif de la forme bien connue :

$$
r . v_{\varphi}=\text { constante }=r^{\prime} \cdot v_{\varphi}
$$

L'équation, valable pour des rapports bidimensionnels et des fluides idéaux, exprime que, lors de sa progression vers l'orifice, le moment cinétique $r . v_{\varphi}$ reste constant et, de manière plus précise, égal au produit de la vitesse de rotation $v_{\varphi}$, par une distance $r^{\prime}$ à l'orifice, produit que l'on appellera «moment cinétique initial ».

Dans le cas des conditions d'écoulement tridimensionnel des fluides réels, la relation donnée dans Téquation ci-dessus n'est plus valable, même pas approximativement. Le travail de frottement absorbe la presque totalité du moment cinétique des fluides visqueux. L'enfoncement de la surface du liquide n'atteint, de ce fait, qu'une valeur finie.

Les équations de Navier-Stokes constituent les équations initiales pour déterminer la variation du moment cinétique dans les fluides visqueux tridimensionnels :

$$
\begin{aligned}
\frac{d v_{r}}{d t}-\frac{v_{\varphi}^{2}}{r}=-\frac{1}{\rho} & \frac{\partial(p+\gamma h)}{\partial r} \\
& +\nu\left(\Delta^{2} v_{r}-\frac{v_{r}}{r^{2}}-\frac{2}{r^{2}} \frac{\partial v_{\varphi}}{\partial \varphi}\right) \\
\frac{d v_{\varphi}}{d t}+\frac{v_{r} \cdot v_{\varphi}}{r}= & -\frac{1}{\rho} \frac{1}{r} \frac{\partial(p+\gamma h)}{\partial \varphi} \\
& +\nu\left(\Delta^{2} v_{\varphi}-\frac{v_{\varphi}}{r^{2}}+\frac{2}{r^{2}} \frac{\partial v_{r}}{\partial \varphi}\right) \\
\frac{d v_{z}}{\partial t}= & -\frac{1}{\rho} \frac{\partial(p+\gamma h)}{\partial z}+\nu\left(\Delta^{2} v_{z}\right)
\end{aligned}
$$

\footnotetext{
* Oberingenieur an Theodor Rehbock-Flusslaboratorium der Technischen Hochschule Karlsruhe.
}

avec les opérateurs :

$$
\begin{aligned}
& \frac{d}{d t}=\frac{\partial}{\partial t}+v_{r} \frac{\partial}{\partial r}+\frac{v_{\varphi}}{r} \frac{\partial}{\partial \varphi}+v_{z} \frac{\partial}{\partial z} \\
& \Delta^{r}=\frac{\partial^{2}}{\partial r^{2}}+\frac{1}{r} \frac{\partial}{\partial r}+\frac{1}{r^{2}} \frac{\partial^{2}}{\partial \varphi^{2}}+\frac{\partial^{2}}{\partial z^{2}}
\end{aligned}
$$

La condition de continuité s'écrit :

$$
\frac{\partial v_{r}}{\partial r}+\frac{v_{r}}{r}+\frac{1}{r} \frac{\partial v_{z}}{\partial \varphi}+\frac{\partial v_{z}}{\partial z}=0
$$

Dans ces équations les abréviations signifient:

$\rho=$ densité du fluide;

$\gamma=$ poids spécifique du fluide;

$p=$ pression;

$\nu=$ viscosité cinématique;

Les coordonnées polaires $r, \varphi$ et la cote $z$ constituent le système de coordonnées.

Les vitesses admettent les trois composantes :

$$
\begin{aligned}
& v_{r}=\text { composante radiale; } \\
& v_{\varphi}=\text { composante de rotation; } \\
& v_{\alpha}=\text { composante verticale. }
\end{aligned}
$$

L'intégration de la deuxième équation de NavierStokes, qui renseigne sur la variation du moment cinématique en chemin ver's l'orifice de sortie, constitue le très intéressant sujet d'un ouvrage publié par H. A. Einstein et Huon Li [1].

A l'intérieur $\left(r \leqq r_{e}\right)$ et à l'extérieur $\left(r \geqq r_{e}\right)$ du cylindre, au-dessus de l'orifice de rayon $r_{e}$ et sous certaines conditions simplificatrices, on obtient pour le rapport du moment cinétique variable au moment cinétique initial $\left(r v_{\varphi} / r^{\prime} v_{\varphi^{\prime}}^{\prime}\right)=\mathrm{RV}_{\varphi}$, les valeurs suivantes :

pour $r \leqq r_{e}$ :

$\mathrm{RV}_{\varphi}=\mathrm{R}_{e} \mathrm{~V}_{\varphi_{e}} \frac{e^{-(\mathrm{A} / 2)\left(\mathrm{R} / \mathrm{R}_{e}\right)^{2}}-1}{e^{-(\mathrm{A} / 2)}-1}$

pour $r \geqq r_{e}$ :

$\mathrm{RV}_{\varphi}=\frac{\left(\mathrm{R}_{e} \mathrm{~V}_{\varphi_{e}}-\mathrm{R}_{e}^{(2-A)}\right)\left(1-\mathrm{R}^{(2-A)}\right)}{1-\mathrm{R}_{e}^{(2-A)}}+\mathrm{R}^{(2-A)}$ 
avec :

$$
\mathrm{R}_{\theta} V_{\varphi_{c}}=\frac{(2-\mathrm{A})\left(e^{-(\Lambda / 2)}-1\right)}{\mathrm{A}\left(1-e^{-(A / 2)} \mathrm{R}_{\left.e^{(A \cdot 2)}\right)+2\left(e^{-(\mathrm{A} / 2)}-1\right)}\right.}
$$

et :

$$
\mathrm{A}=\frac{\mathrm{Q}_{e}}{2 \pi h \nu}
$$

$r_{e}, v_{\varphi_{e}}$ est le moment cinétique à une distance $r_{C}$ de l'axe de l'orifice, $Q_{e}$ le débit à l'orifice et $h$ la hauteur d'eau au-dessus du fond.

Les suppositions fondamentales faites, pour aboutir aux équations (2.1) et (5), par Einstein et Huon Li sont en substance les suivantes :

$1^{\circ}$ En comparaison des vitesses horizontales, les vitesses verticales sont secondaires;

$2^{\circ}$ La répartition des vitesses dans la section de l'orifice est constante :

$$
v_{e}=\frac{\mathrm{Q}_{e}}{\pi r_{c}^{2}}=\text { constante. }
$$

Le débit à l'intérieur d'un cylindre déterminé $\left(r<r_{e}\right)$ se réduit de la quantité d'eau s'écoulant extérieurement à ce cylindre, et prend la valeur :

$$
\mathrm{Q}_{r}=\frac{\mathrm{Q}_{e} \cdot r^{2} \pi}{r_{0}^{2} \pi}
$$

Correspondant à cela et en raison de la continuité, la vitesse radiale moyenne diminue aussi audessus de l'orifice jusqu'à la valeur :

$$
v_{r}=\frac{\mathrm{Q}_{c} \cdot r^{2}}{2 \pi \mathrm{r} \cdot h \cdot r_{\epsilon}^{2}}=\frac{\mathrm{Q}_{e} \cdot r}{2 \pi \cdot h \cdot r_{e}^{2}}
$$

Le but de ce travail-ci est de calculer, par intégration de la première équation de Navier-Stokes, la forme de la surface libre, et, de là, formuler les conditions de similitude du vortex.

\section{La surface libre du vortex.}

Sous considération des suppositions ci-dessus, la première équation de Navier-Stokes prend la forme :

$$
\begin{aligned}
v_{r} \frac{\partial v_{r}}{\partial r}-\frac{v_{\varphi}^{2}}{r}=- & \frac{1}{\rho} \frac{\partial(p+\gamma h)}{\partial r} \\
& \left(\nu \frac{\partial^{2} v_{r}}{\partial r^{2}}+\frac{1}{r} \frac{d v_{r}}{\partial r}-\frac{v_{r}}{r^{2}}\right)
\end{aligned}
$$

On obtient alors, tout d'abord par intégration :

$$
\frac{v_{r}^{2}}{2}-\int \frac{v_{\varphi}^{2}}{r} d r=-\frac{p+\gamma h}{\rho}+\mathrm{C}
$$

On a en efret :

$$
\int v_{r} \frac{\partial v_{r}}{\partial r} d r=\frac{v_{r}^{2}}{2}
$$

Et les équations pour la vitesse radiale et ses dérivées partielles s'écrivent:

$$
\begin{array}{rcc} 
& \text { pour } r \geqq r_{e} & \text { pour } r \leqq r_{e} \\
v_{r}= & \frac{\mathrm{Q}_{e}}{2 \pi \cdot h \cdot r} & \frac{\mathrm{Q}_{e} \cdot r}{2 \pi \cdot h \cdot r_{e}{ }^{2}} \\
\frac{\partial v_{r}}{\partial r}= & \frac{\mathrm{Q}_{e}}{2 \pi \cdot h \cdot r^{2}} & \frac{\mathrm{Q}_{e}}{2 \pi \cdot h \cdot r_{e}{ }^{2}} \\
\frac{\partial^{2} v_{1}}{\partial r^{2}}= & \frac{\mathrm{Q}_{c}}{\pi \cdot h \cdot r^{3}} & 0
\end{array}
$$

L'intégrale :

$$
\int\left(\frac{\partial^{2} v_{r}}{\partial r^{2}}+\frac{1}{r} \frac{\partial r^{2}}{\partial r}-\frac{v_{r}}{r^{2}}\right) d r
$$

devient alors, pour $r \geqq r_{c}$ et $r \leqq r_{e}$, égale à zéro.

Dans le domaine $r \geqq r_{c}$, on obtient le moment cinétique à partir de la deuxième expression de l'équation (2.1).

Avec :

$$
\mathrm{B}=\frac{\mathrm{R}_{r} \mathrm{~V}_{\varphi_{e}}-\mathrm{R}_{r}{ }^{(2-A)}}{1-\mathrm{R}_{e}{ }^{(2-A)}}
$$

on transforme comme suit l'équation :

$r \cdot v_{\varphi}=r^{\prime} \cdot v_{\varphi}^{\prime} \mathrm{B}-r^{\prime} v_{\varphi}^{\prime} \mathrm{BR}^{(2-A)}+r^{\prime} v^{\prime}{ }_{\varphi} \mathrm{R}^{(2-A)}$

ou bien encore :

$$
r . v_{\varphi}=r^{\prime} v_{\varphi}^{\prime}\left[\mathrm{B}-(\mathrm{B}-1) \mathrm{R}^{(2-A)}\right]
$$

Pour l'intégrale $\int\left(v_{\iota_{0}}^{2} / r\right) d r$ dans l'équation (1.2), on obtient donc l'expression suivante :

$\int \frac{v_{\phi}^{2}}{r} d r=\int \frac{r^{\prime 2} v_{\varphi}^{\prime}\left[\mathrm{B}-(\mathrm{B}-1) \mathrm{R}^{(2-A)}\right]^{2}}{r^{3}} d r$

ou bien, sous une autre forme :

$$
\begin{gathered}
\int \frac{v_{\varphi}^{2}}{r} d r=v_{\varphi^{2}}^{2} \\
\quad\left[\mathrm{~B}^{2} \mathrm{R}-3-2 \mathrm{~B}(\mathrm{~B}-1) \mathrm{R}-(1+\mathrm{A})\right. \\
\left.+(\mathrm{B}-1)^{2} \mathrm{R}^{(1-2 \mathrm{~A})}\right] d \mathrm{R}
\end{gathered}
$$

L'intégration donne:

$$
\begin{aligned}
& \int \frac{v_{\varphi}^{2}}{r} d r=v_{\varphi}^{\prime}\left[-\frac{\mathrm{B}^{2} \mathrm{R}^{-2}}{2}\right. \\
& \left.\quad+\frac{2 \mathrm{~B}(\mathrm{~B}-1) \mathrm{R}-\mathrm{A}}{\mathrm{A}}-\frac{(\mathrm{B}-1)^{2} \mathrm{R}^{2(1-A)}}{2(1-\mathrm{A})}\right]
\end{aligned}
$$

Pour l'eau, les valeurs $A<10$ sont d'intérêt secondaire. Comme on peut le prouver par l'équation (2.1) (fig. 1) et comme on l'a constaté à la lueur des résultats expérimentaux [2], les pertes par viscosité à l'extérieur du cylindre de rayon $r=r_{\theta}$ sont cependant, dans le cas $\mathrm{A}>10$, négligeables. Le rapport $\mathrm{R}_{e} \mathrm{~V}_{\varphi}$, tend, pour $A>10$, vers la valeur limite 1 . L'opérateur $B$ (équation 8), tend lui aussi vers la valeur 1 , avec $R_{e} V_{\varphi_{e}}=1$.

Avec $\mathrm{B}=1$, l'équation (9.2) devient :

$$
\int \frac{v_{\varphi}^{2}}{r} d r=-\frac{v_{\varphi}^{\prime}{ }^{2} R^{-2}}{2}=\frac{v_{\varphi}^{\prime 2}}{2} \frac{r^{\prime 2}}{r^{2}}
$$

Dans le domaine $r \leqq r_{\ell}$, on obtient le moment cinétique par la première expression de l'équation (2.1). 
Avec les opérateurs :

$\mathrm{D}=\frac{r_{e}{ }^{2} v_{\varphi_{e}{ }^{2}}}{\left(e^{-(\mathrm{A} / 2)}-1\right)^{2}} ; \quad \mathrm{C}=-\frac{\mathrm{A}}{2}$ et $\mathrm{R}^{\prime}=\frac{\mathrm{R}}{\mathrm{R}_{e}}=\frac{r_{c}}{r}$

on obtient pour l'intégrale $\int\left(v_{\varphi}^{2} / r\right) d r$ la relation :

$$
\begin{aligned}
\int \frac{v_{\varphi}{ }^{2}}{r} d r= & \mathrm{D} \int \frac{\left(e^{\mathrm{Cr} \mathrm{r}^{\prime 2}}-1\right)^{2}}{r^{3}} d r \\
& =\frac{\mathrm{D}}{r_{\sigma^{2}}} \int \frac{e^{2 \mathrm{CR}^{\prime 2}}-2 e^{\mathrm{CR} \mathrm{R}^{\prime 2}}+1}{\mathrm{R}^{\prime 3}} d \mathrm{R}^{\prime}
\end{aligned}
$$

On ne peut pas, sous cette forme, résoudre l'intégrale. On la développe alors en série, puis l'on intègre membre par membre:

$\int \frac{v_{\varphi}^{2}}{r^{2}} d r=\frac{D}{r_{e}^{2}}$

$\int\left[\frac{1+\frac{\left(2 \mathrm{CR}^{\prime 2}\right)^{1}}{1 !}+\frac{\left(2 \mathrm{CR}^{\prime 2}\right)^{2}}{2 !}+++\frac{\left(2 \mathrm{CR}^{\prime 2}\right)^{n}}{n !}+++}{\mathrm{R}^{\prime 3}}\right.$

$\left.\frac{-2\left(1+\frac{\left(\mathrm{CR}^{\prime 2}\right)^{1}}{1 !}+\frac{\left(\mathrm{CR}^{\prime 2}\right)^{2}}{2 !}+++\frac{\left(\mathrm{CR}^{\prime 2}\right)^{n}}{n !}+++\right)+1}{\mathrm{R}^{\prime 3}}\right] d \mathrm{R}^{\prime}$

On peut rassembler les termes du développement de l'équation (11.1):

$\int \frac{v_{\varphi}^{2}}{r} d r=\frac{\mathrm{D}}{r_{e^{2}}^{2}} \int$

$\frac{\frac{2 \mathrm{C}^{2} \mathrm{R}_{4}^{\prime}}{2 !}+\frac{6 \mathrm{C}^{2} \mathrm{R}^{\prime 6}}{3 !}+++\frac{\left(2^{n}-2\right) \mathrm{C}^{n} \mathrm{R}^{\prime 2 n}}{n !}+++}{\mathrm{R}^{3}} d \mathrm{R}^{\prime}$

En intégrant membre par membre, on obtient :

$$
\begin{aligned}
& \left.\int \frac{v_{\varphi}{ }^{2}}{r} d r=\frac{v_{\varphi}{ }^{2}}{(e-(\mathrm{A} / 2)}-1\right)^{2} \\
& \quad\left(\frac{2(\mathrm{~A} / 2)^{2}\left(r / r_{c}\right)^{2}}{2.2 !}-\frac{6(\mathrm{~A} / 2)^{3}\left(r / r_{e}\right)^{4}}{4.3 !}+-+\right. \\
& \frac{\left(2^{n}-2\right)[-(\mathrm{A} / 2)]^{n}\left(r / r_{c}\right)^{(2 n-2)}}{(2 n-2) n !}+\cdots+
\end{aligned}
$$

L'exploitation de la relation (11.3) est rendue difficile par la convergence particulièrement faible de la série alternante. Comme, en général, on devrait introduire dans chacun des membres des grandes valeurs de $A(>10)$, l'exploitation numérique ne serait alors possible qu'à la calculatrice électronique.

Dans ce qui suit, on développe une méthode graphique pour l'exploitation de l'équation. A est la seule grandeur hydraulique sans dimensions contenue dans les membres de la série. Le rapport sans dimensions $r / r_{c}$ tient compte de la géométrie de l'orifice. On peut donc, en intégrant l'équation (11.3) graphiquement par quelques valeurs de $A$, déterminer la somme $\Sigma$ des membres dans l'équation à partir de l'équation (11.3) résolue par rapport à $\Sigma$.

$$
\Sigma=\int \frac{v_{\varphi}^{2}}{r} \frac{d r\left(e^{-(A / 2)}-1\right)^{2} r_{e}^{2}}{r_{c}^{2} v_{\varphi \varphi^{2}}{ }^{2}}
$$

Sur le nomogramme de la figure 1 , on a porté le rapport du moment cinétique instantané au moment cinétique sur le eylindre de rayon $r_{i}$, en fonction du rapport sans dimensions $r / r_{e}$, pour différentes valeurs de $\mathrm{A}$.

\begin{tabular}{|c|c|c|c|}
\hline & A & $r_{0}$ & $r_{c} v_{\varphi \varphi}$ \\
\hline 1 & 5 & 1 & 40 \\
\hline 2 & 10 & 1 & 20 \\
\hline 3 & 15 & 1 & 20 \\
\hline 4 & 20 & 1 & 16 \\
\hline 5 & 30 & 1 & 12 \\
\hline 6 & 50 & 1 & 10 \\
\hline 7 & 100 & 1 & 5 \\
\hline
\end{tabular}

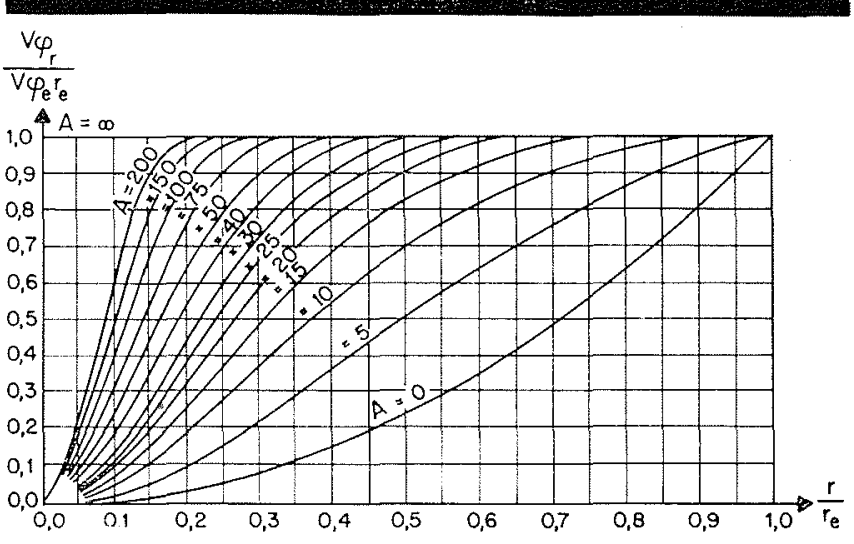

1/ Diminution du moment cinétique.

Reduction of linetic moment.

L'intégration graphique a été réalisée dans les cas suivants :

Le résultat de l'intégration graphique est exploité sur le nomogramme de la figure 2.

Le résultat de l'intégration de la première équation différentielle de Navier-Stokes s'écrit alors sous la forme condensće :

pour $r \geqq r_{e}$ :

$$
\frac{v_{r}^{2}}{2}+\frac{v^{\prime} \varphi^{2}}{2} \frac{r^{\prime 2}}{r^{2}}=-\frac{p+\gamma h}{\rho}+\mathrm{C}_{1}
$$

pour $r \leqq r_{e}$ :

$$
\frac{v_{r}^{2}}{2}-\frac{v_{\varphi_{c}}^{2} \cdot \Sigma}{(e-(A / 2)-1)^{2}}=-\frac{p+\gamma h}{\rho}+\mathrm{C}_{2}
$$

Les deux équations constituent les équations énergétiques du vortex. Pour $p=0, h$ donne la surface libre $d u$ vortex. La constante $C_{1}$ sobtient à partir de la condition limite :

$$
r=r^{\prime} \rightarrow \frac{\mathrm{C}_{1}}{g}=\frac{v_{r}^{\prime}}{2 g}+\frac{v_{\varphi}^{\prime}}{2 g}+h=\mathrm{H}
$$

$\mathrm{C}_{1} / g$ est donc la hauteur d'énergie au point initial de la rotation. Dans le cas $r \leqq r_{e}$, on obtient pour $r=0$ :

$$
\mathrm{C}_{2}=g \cdot h_{0} \text {. }
$$

$\mathrm{C}_{2} / \mathrm{g}$ est alors l'ordonnée de la pointe du tourbillon. Avec cela, les équations de la surface libre du vortex s'écrivent en définitive :

pour $r \geqq r_{o}$ :

$$
h=\mathrm{H}-\frac{\nu^{\prime 2}}{2 g}-\frac{\left[v_{\varphi}^{\prime} \cdot\left(r^{\prime} / r\right)\right]^{2}}{2 g}
$$

pour $r \leqq r_{e}$ :

$$
h=h_{0}-\frac{v_{r}^{2}}{2 g}+\frac{v_{\varphi e^{2}}}{g\left(1-e^{-(\Lambda / 2)}\right)^{2}} \cdot \Sigma
$$




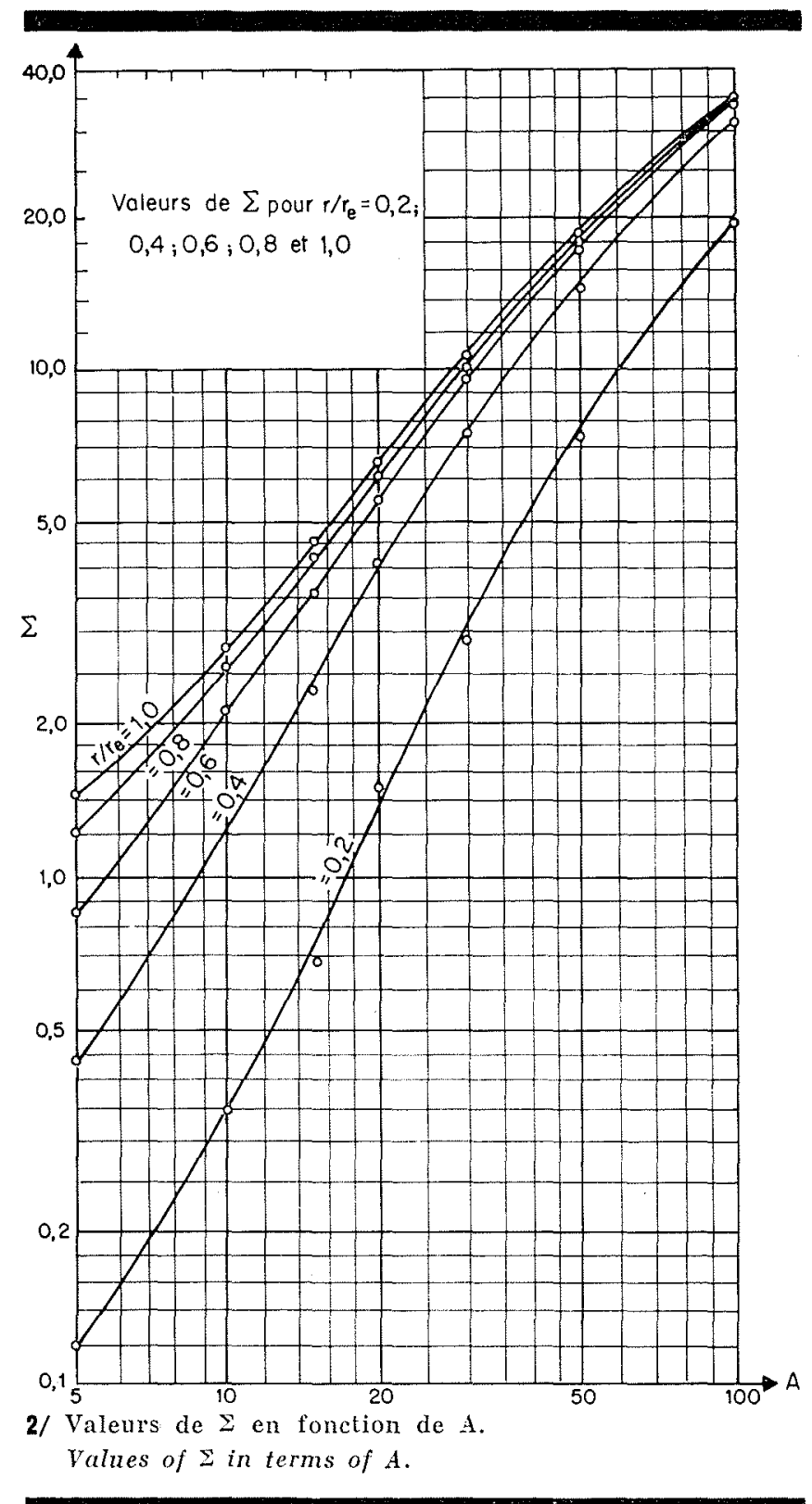

La répartition des vitesses de l'écoulement radial est précisée dans les équations suivantes:

pour $r \geqq r_{e}$ :

pour $r \leqq r_{\epsilon}$ :

$$
v_{r}=\frac{\mathrm{Q}_{e}}{2 \pi \cdot h \cdot r}
$$

(voir équation 7).

$$
v_{r}=\frac{Q_{e} \cdot r}{2 \pi \cdot h \cdot r_{c}^{2}}
$$

Pour vérification des équations de la surface libre, en particulier quand $r \leqq r_{c}$, on peut d'abord constater qu'aux limites $A=0$ et $A=\infty$, les équations correspondent aux lois ici valables. Si l'on développe en série, tant la fonction :

$$
\sum\left(A_{i} \frac{r}{r_{e}}\right)
$$

que l'expression $\left(1-e^{-(A / 2)}\right)^{2}$, on trouve :
Si l'on divise haut et bas par $A^{2}$, le passage à la limite $\mathrm{A} \rightarrow 0\left(\mathrm{Q}_{e}=0, v_{r}=0\right)$ donne :

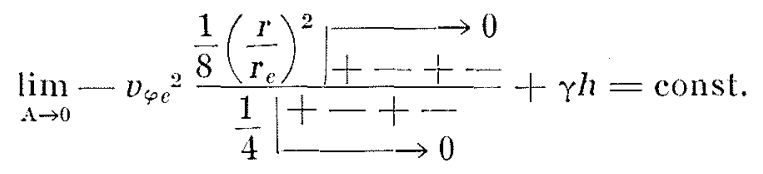

ou bien :

$$
\left.-\frac{1}{2} v_{\varphi \varphi^{2}}{ }^{2} \omega\right)^{2}+\gamma h=\text { const. }
$$

$\omega=v_{\varphi_{e}} / r_{e}$ est la vitesse angulaire. L'équation 13 est bien connue (parabole du deuxième ordre) et représente la dépression de la surface sous l'effet d'un mouvement tourbillonnaire invariable.

Dans le cas d'un fluide idéal, $A=\infty(\nu=0)$, l'équation 11 prend alors la forme :

$$
\int \frac{v_{\varphi}^{2}}{r} d r=\int \frac{r_{e}{ }^{2} v_{\varphi e^{2}}}{r^{3}} d r=-\frac{r_{e}{ }^{2} v_{\varphi_{e}}{ }^{2}}{2 r^{2}}
$$

et l'équation de la surface libre s'écrit :

$$
h=\mathrm{H}-\frac{v_{i}^{2}}{2 g}-\frac{v_{\varphi_{e}}{ }^{2}}{2} \frac{r_{e}^{2}}{r_{c}}
$$

L'équation 14 est l'équation bien connue de la surface libre dans le cas d'un mouvement de rotation d'un fluide sans frottements.

Pour les valeurs finies de $A$, on a pu vérifier les résultats théoriques par des expériences réalisées en 1940 dans les laboratoires hydrauliques de Karlsruhe [2]. On compare dans la figure 3 une partie des résultats photographiques expérimentaux avec les valeurs théoriques.

On constatait en général, que pour de grandes valeurs du moment cinétique initial $r^{\prime} v_{\varphi}^{\prime}$, les profondeurs du creux trouvées expérimentalement étaient légèrement supérieures aux valeurs théoriques. La raison de cet écart devrait être la non-

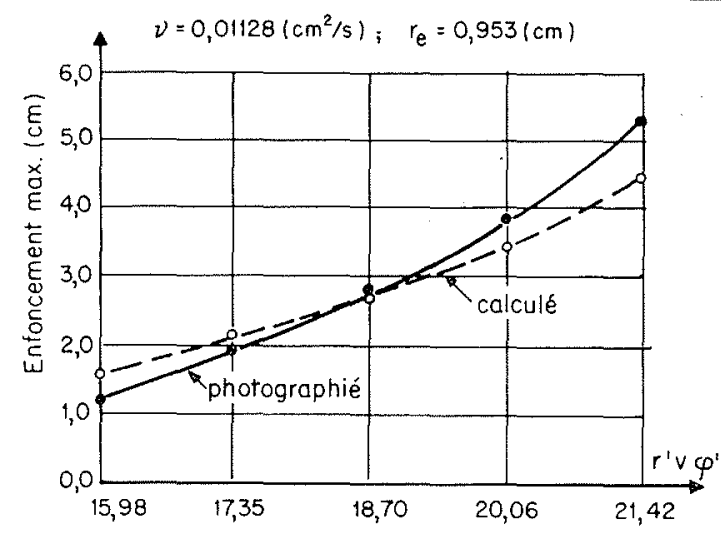

3/ Enfoncement maximal du vortex.

$$
\text { Vortex becoming deeper. }
$$

$$
\frac{v_{r}^{2}}{2}-v_{\varphi_{e}}{ }^{2} \frac{\frac{2(\mathrm{~A} / 2)^{2}\left(r / r_{e}\right)^{2}}{2.2 !}-\frac{6(\mathrm{~A} / 2)^{3}\left(r / r_{e}\right)^{4}}{4.3 !}+-\frac{\left(2^{n}-2\right)[-(\mathrm{A} / 2)]^{n}\left(\mathrm{r} / r_{e}\right)^{(2 n-2)}}{(2 n-2) n !}-\frac{1}{4}-\mathrm{A}^{2}-\frac{1}{8} \mathrm{~A}^{3}+--\frac{\mathrm{A}^{n}\left(2^{n}-2\right.}{2^{n} \cdot n !}-+-}{-}+\gamma^{h}=\text { const. }
$$


coïncidence des conditions marginales théoriques et expérimentales.

Tandis que la théorie suppose une répartition constante de la vitesse à la hauteur $h$, on doit admettre que, lors des expériences, la vitesse, à cause des frottements au fond, a augmenté dans les couches supérieures qui sont déterminantes de la forme du tourbillon. La raison de l'écart dans le cas de petites valeurs $r^{\prime} v_{\varphi}^{\prime}$ est vraisemblablement l'influence de la tension superficielle à la pointe du tourbillon.

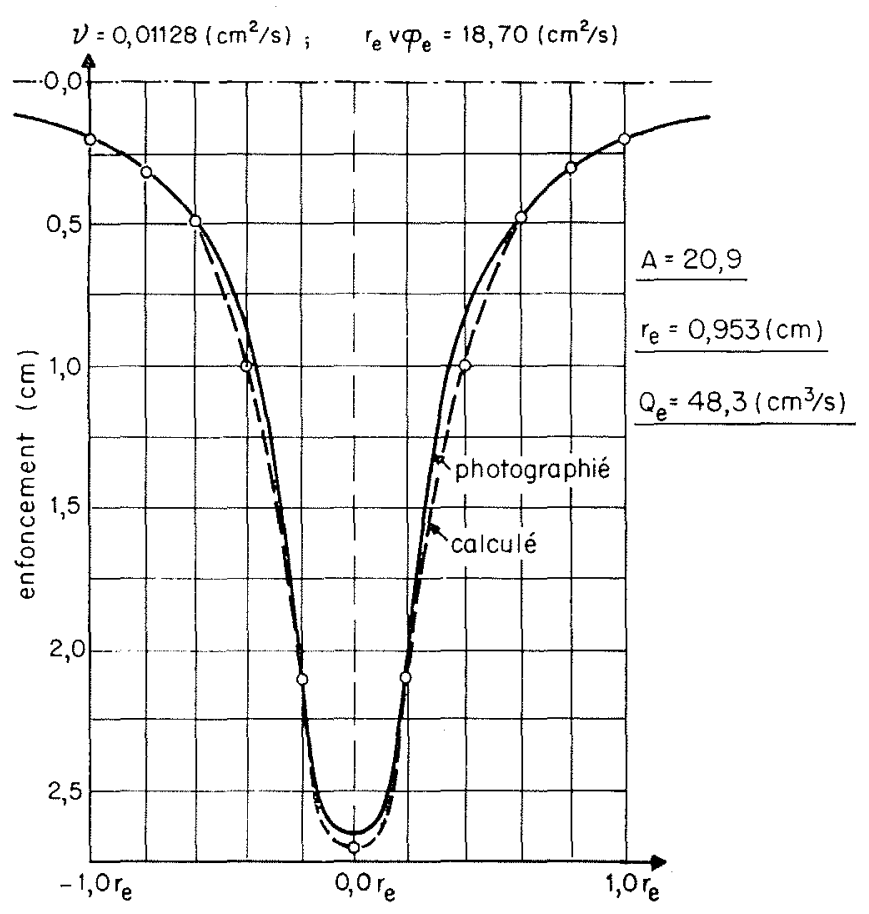

4) Profil du vortex creux.

Calculated and photographed eddy contours.

La figure 4 montre les contours d'un tourbillon calculé, et d'un tourbillon photographié.

\section{Les conditions de similitude du vortex.}

La valeur de la vitesse dans l'écoulement radial n'exerce sur la surface libre d'une influence secondaire. La valeur maximale de la dépression est complètement indépendante de la vitesse de l'écoulement radial, lorsque la vitesse radiale devient nulle à la pointe du tourbillon. De ce fait, on ne tiendra pas compte de la vitesse radiale dans ce qui suit.

Les rapports des hauteurs (profondeurs) de dépression $(\mathrm{H}-h) / r_{e}$ pour $r \geqq r_{\theta}$ et $\left(h-h_{0}\right) / r_{e}$ dépendent des paramètres suivants.

pour $r \geqq r_{c}: v_{\varphi}^{\prime}, r^{\prime}, r, g$

pour $r \leqq r_{c}: v_{\varphi_{e}}, v_{r_{o}}, r_{e}, r, g, \nu$
On peut alors développer les combinaisons sans dimensions suivantes :

pour $r \geqq r_{e}:$

$$
\frac{r^{\prime}}{r} ; \frac{v_{\varphi}^{\prime}{ }^{2}}{g \cdot r^{\prime}}
$$

pour $r \leqq r_{e}$ :

$$
\frac{r}{r_{e}} ; \frac{v_{\varphi_{e}}{ }^{2}}{g \cdot r_{e}} ; \frac{v_{r_{a}} \cdot r_{e}}{\nu}
$$

Si l'on tient compte que :

$$
\frac{v_{\varphi}^{\prime}{ }^{2}}{g \cdot r^{\prime}} \text {, et } \frac{v_{\varphi_{e}}^{2}}{g \cdot r_{e}}
$$

sont les nombres de Froude du mouvement de rotation, et que $v r_{\theta}, r_{c} / \nu$ est le nombre de Reynolds du mouvement radial, le système (15.1) exprime alors, qu'à côté des grandeurs qui caractérisent la géométrie du système $\left(r^{\prime} / r\right)$, la surface libre ne dépend dans le cas $r \geqq r_{e}$ que du nombre de Froude du mouvement de rotation. Dans le cas $r \leqq r_{e}$, le nombre de Reynolds du mouvement exerce aussi, en plus des grandeurs géométriques $\left(r / r_{e}\right)$ et du nombre de Froude de la rotation, une influence sur la forme de la surface libre:

$$
\begin{aligned}
& \text { pour } r \geqq r_{e}: \\
& \qquad \frac{\mathrm{H}-h}{r_{e}}=f\left(\frac{r^{\prime}}{r} ; \mathscr{F}_{\mathrm{rot}}\right)
\end{aligned}
$$

pour $r \leqq r_{\varepsilon}$ :

$$
\frac{h-h_{0}}{r_{e}}=f\left(\frac{r}{r_{e}} ; \mathscr{Y}_{\mathrm{rot}} ; \mathcal{R}_{\mathrm{rud}}\right)
$$

Pour la similitude des mouvements tourbillonnaires réels et en modèle réduit, on a les conditions suivantes:

$$
\begin{aligned}
& \text { pour } r \geqq r_{e} \text { : } \\
& \left(\frac{r^{\prime}}{r}\right)_{\mathrm{M}}=\left(\frac{r^{\prime}}{r}\right)_{\mathrm{N}} ; \mathscr{F}_{\mathrm{rot} \mathrm{M}}=\mathscr{H}_{\operatorname{rot} \mathrm{N}} \\
& \text { pour } r \leqq r_{e} \text { : } \\
& \left(\frac{r}{r_{e}}\right)_{\mathrm{M}}=\left(\frac{r}{r_{e}}\right)_{\mathrm{N}} ; \mathscr{F}_{\mathrm{rot} \mathrm{M}}=\mathscr{F}_{\mathrm{rot} \mathrm{N}} ; \mathcal{R}_{\mathrm{rad} \mathrm{M}}=\mathfrak{R}_{\mathrm{rad} N}
\end{aligned}
$$

où les indices $\mathrm{M}, \mathrm{N}$ caractérisent les valeurs du modèle et celles de la nature. Ces conditions de similitude peuvent être remplies pour des propriétés analogues du fluide dans le modèle et dans la réalité.

Le nombre de Reynolds du mouvement radial, $\mathcal{O}_{\text {rad }}$ extérieurement et :

$$
\mathcal{R}_{\mathrm{rad} r_{0}}=v_{r_{\mathrm{e}}} r_{f} / \nu
$$

sur le cylindre de rayon $r_{e}$, est identique à la valeur A. Entre $\mathscr{F}^{\prime}$ rotr et $\mathscr{g} r$ rot $r_{e}$, et à cause de la relation $r_{c} v_{\varphi_{e}}=r^{\prime} v_{\varphi}^{\prime}$ (dans le cas $\mathrm{A}>10$, voir l'équation 9.3), on obtient le résultat suivant:

$$
\mathscr{F H}_{\text {rot } r^{\prime}}=\mathscr{F}_{\operatorname{rot}} v_{e} \frac{r_{e}^{3}}{T^{\prime 3}}
$$

En tenant compte de l'équation 1.4 et du fait 
que $v_{r}^{2} / 2 g$ est négligeable, les conditions de similitude s'énoncent :

$$
\begin{aligned}
& \text { pour } r \geqq r_{e}: \\
& \qquad \frac{\mathrm{H}-h}{r_{e}}=\frac{r_{r}{ }^{2}}{2 r^{2}} \mathscr{\mathscr { Y }} \operatorname{rot} r_{c}
\end{aligned}
$$

pour $r \leqq r_{e}$ :

$$
\frac{h-h_{0}}{r_{c}}=\mathscr{g}_{\operatorname{rot} r_{e}} \frac{\sum\left[\mathcal{R} \operatorname{ma} r_{e} ;\left(r / r_{e}\right)\right]}{\left(1-e^{-(A / 2))^{2}}\right.}
$$

En général, on peut, comme il a déjà été mentionné plusieurs fois, mettre les équations sous la forme suivante :

$$
\begin{aligned}
& \text { pour } r \geqq r_{o}: \\
& \qquad \frac{\mathrm{H}-h}{r_{\theta}}=\frac{r_{\mathrm{r}}{ }^{2}}{2 r^{2}} \mathscr{J}_{\mathrm{rot} .} .
\end{aligned}
$$

pour $r \leqq r_{e}$ :

$$
\frac{h-h_{0}}{r_{e}}=\mathscr{G H}_{\text {rot } r_{e}} \cdot \Sigma\left(\mathcal{A}_{\operatorname{rad} r_{e}} \frac{r}{r_{c}}\right)
$$

Lorsque les valeurs de A ou $\mathcal{R}_{\text {rad }}$, deviennent très grandes, c'est-à-dire $1--c^{-(A / 2)} \sim 1$, les équations (15.5) sont les conditions nécessaires pour la similitude des surfaces libres. La fonction :

$$
\Sigma\left(\mathcal{R} \operatorname{rad} r_{c} ; \frac{r}{r_{e}}\right)
$$

est représentée sur la figure 2 .

La difficulté de l'expérimentation en laboratoire réside dans l'existence de deux conditions différentes pour le modèle réduit pour un problème expérimental unique.

Une similitude dans le domaine $r \geqq r_{c}$ est nécessaire pour comprendre l'influence décisive de la géométrie sur le moment cinétique. La similitude dans le domaine $r \leqq r_{e}$ est utile pour garantir dans le modèle une forme du tourbillon conforme à celle de la nature.

La manière dont on solutionne ces problèmes de la facon la plus opportune, dans la réalisation des modèles, varie de cas en cas. Si l'influence de la géométrie est décisive (fig. 5, forme 1), la technique expérimentale suivante est avantageuse :

$1^{\circ}$ toutes les grandeurs sont converties d'après la loi de similitude de Froude (également dans le

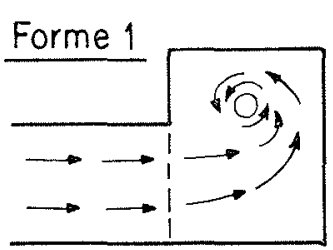

Forme 2
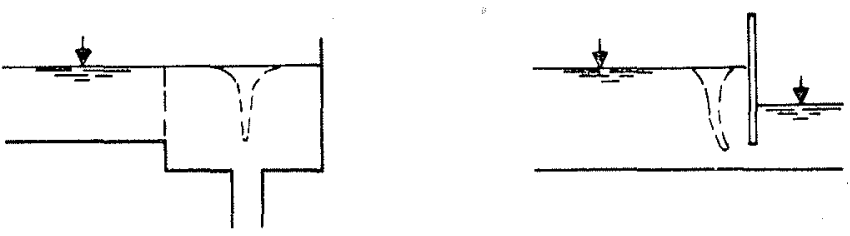

5/ domaine $r \leqq r_{e}$ ), et les inconnues sont trouvées sur le modèle;

$2^{\circ}$ à l'aide de l'équation 15.5 on détermine le nom-

\begin{tabular}{|c|c|c|}
\hline & $\begin{array}{l}\text { VALEURS } \\
\text { RÉELLES }\end{array}$ & $\begin{array}{l}\text { Grandetns } \\
\text { RÉDUITES } \\
\text { DU MODĖLE }\end{array}$ \\
\hline Rayon $r_{e}, \ldots \ldots$ (cm) & 5 & 1 \\
\hline Hauteur d'eau. ......(cm) & 100 & 20 \\
\hline Viscosité & & \\
\hline $\begin{array}{cc}\text { cinématique. } & \left(\mathrm{cm}^{2} / \mathrm{s}\right) \\
\text { Débit d'eau. } . & \left(\mathrm{cm}^{3} / \mathrm{s}\right)\end{array}$ & $\begin{array}{l}0,012 \\
755\end{array}$ & $\begin{array}{l}0,012 \\
13,3\end{array}$ \\
\hline Valeur A. . . . . . . . . & 100 & 8,95 \\
\hline$\Sigma($ de la fig. 2$) \ldots \ldots \ldots$ & 36 & 2,5 \\
\hline
\end{tabular}
bre $\mathscr{Y}_{\text {rot }}$ au point $r=r_{\varepsilon}$;

$3^{\circ}$ la valeur A pour les rapports réels est tirée du nomogramme de la figure 2;

$4^{\circ}$ le rapport réel $\left(h-h_{0}\right) / r_{e}$ se calcule à partir de l'équation 15.5.

EXEMPLE :

Le rapport d'enfoncement de la surface dans la maquette atteindrait 0,5 . De là, on calcule le nombre $\mathscr{g}_{\text {rot }}$ à partir de l'équation 15.5.

$$
\mathscr{F}_{\mathrm{rot}}=\frac{\left[\left(h-h_{0}\right) / r_{e}\right]_{\mathrm{M}}}{r_{c \mathrm{M}} \cdot \Sigma_{\mathrm{M}}}=0,5 / 2,5=0,2
$$

Le rapport réel d'enfoncement de la surface $\left(h-h_{0}\right) / r_{c}$ devient avec $\mathscr{F}_{\text {rot }}=0,2$ :

$$
\left(h-h_{0}\right) / r_{c}=0,2 \Sigma_{\mathrm{N}}=7,2
$$

Dans le cas où l'influence de la géométrie sur

\begin{tabular}{|c|c|c|}
\hline & $\begin{array}{l}\text { VALEURS } \\
\text { RÉDUT'TES }\end{array}$ & $\begin{array}{l}\text { VaLEuRs } \\
\text { RÉELLES }\end{array}$ \\
\hline$r_{e} \ldots \ldots \ldots \ldots \ldots \quad(\mathrm{cm})$ & 5 & 1 \\
\hline$h \ldots \ldots \ldots \ldots \ldots(\mathrm{cm})$ & 100 & 20 \\
\hline v. $\ldots \ldots \ldots \ldots,\left(\mathrm{cm}^{2} / \mathrm{s}\right)$ & 0,012 & 0,012 \\
\hline $\mathrm{Q}_{e} \ldots \ldots \ldots\left(\mathrm{cm}^{3} / \mathrm{s}\right)$ & 755 & $?$ \\
\hline A. . . . . . . . . & 100 & 100 \\
\hline$\Sigma \ldots \ldots \ldots \ldots \ldots \ldots$ & 36 & 36 \\
\hline
\end{tabular}
le moment cinétique est secondaire, et aussi en particulier quand le domaine $r \leqq r_{e}$ n'est pas clairement limité (fig. 5, forme 2), la condition de similitude pour $r \leqq r_{e}$ peut également être prise en considération en dehor's du domaine $r \leqq r_{c}$. Le rapport d'enfoncement est alors identique dans le cas de la maquette comme dans celui de la réalité.

WÊME EXEMPLE :

Le débit d'eau dans la maquette se calcule à partir de l'équation en A (6) :

$$
\mathrm{Q}_{e}=100.2 \pi .0,012.20=151 \mathrm{~cm}^{3} / \mathrm{s}
$$

L'exemple montre que sous l'hypothèse d'un nombre $\mathscr{W}_{\text {rot }}$ identique dans la nature et la maquette, on doit choisir pour les débits et vitesses dans la maquette, des valeur's beaucoup plus grandes que celles données par la loi de similitude de Froude. Ces faits ont été constatés dans des expé- 
riences indépendantes les unes des autres, menées par Denny et Joung [3], Iversen [4] et Fraser [5]. Les quatre auteurs sont unanimes à rapporter que la loi de Froude donne des enfoncements de la pointe du tourbillon trop faibles. Denny et Joung conseillent de choisir dans les expériences sur la maquette, des vitesses beaucoup plus grandes correspondant à peu près aux valeurs réelles, et de ne pas dépasser le rapport de réduction de $1 / 16$.

Si l'on applique cette règle empirique dans l'exemple calculé ci-dessus, on obtient les rapports suivants :

— vitesse à l'entrée :

$$
v_{c}=\frac{Q \mathrm{~N}}{r_{0 \mathrm{~N}}{ }^{2} \pi}=9,62 \mathrm{~cm} / \mathrm{s}
$$

- débit à l'orifice dans la maquette :

$$
Q_{0 \mathrm{I}}=9,62 \cdot r_{e \mathrm{~N}}^{2} \cdot \pi=30,2 \mathrm{~cm}^{3} / \mathrm{s} \text {; }
$$

- valeur A dans la maquette :

$$
A_{\mathrm{XI}}=\frac{\mathrm{Q}_{\mathrm{H}}}{2 \pi \cdot h \cdot \mathrm{v}}=20,1 \text {; }
$$

$-\Sigma_{\mathrm{Xr}}($ de la fig. 2$)=7,0$;

- $\mathscr{J}_{\mathrm{rot} \lambda}=\mathscr{\mathscr { H }}_{\mathrm{rot} \mathrm{X}} .5=1,0$.
Le rapport d'enfoncement d'après la règle empirique devient :

$$
\left(h-h_{0}\right) / r_{e}=\mathscr{H}_{\operatorname{rot} \mathrm{II}} . \Sigma_{\mathrm{MI}}=7,0
$$

Ce rapport atteignait d'après l'équation 15.5 , pour le même exemple, la valeur 7,2 .

\section{References}

[1] Eanstein et Huon Li. -- Le vortex permanent dans un fluide l'éel, La Houlle Blanche, août 1955.

[2] A. Hoefforex. - Beitrag zur Kenntnis der Wirbelbewegung in zähen Flüssigkeiten. Karlsruhe, 1940.

[3] DENNY and Yousg. - The prevention of vortices and swirls at intakes. $I A H R$, 7th general meeting, 1957.

[4] Ivensen. - Studies of submergence requirements of high-specifte speed pumps. Trans. ASME, vol. 75, n० 4 .

[5] Fraser. - Hydraulic problems encountered in intake structures of vertical wet-pit pumps and methods leading to their solution. Trans. ASME, vol. $75, \mathrm{n}^{\circ} 4$.

\section{Abstract \\ The free surface and conditions of similarity for a drain vortex \\ by G. Holtorff *}

\section{Introduction.}

Stable flow above a drain in shallow water is only possible if a vortex motion of the well-known form $v_{\varphi} . r=$ constant is superposed on it. This relation indicates that the moment of momentum is constant on its way to the drain. In the case of real fluids the moment of momentum is almost entirely consumed by friction losses. For the decay of moment of momentum approaching the drain a solution was developed by $H$. A. Einstein and Huon Li from the second Navier-Stokes equation (eq. 2). The results obtained by integrating the second Navier-Stockes equation under some simplifying assumptions are given in equations 2,1; 5 and 6 . Essentially the following assumptions were made :

1. Negligible average vertical velocity components;

2. Uniform velocity distribution at the drain opening.

In equation 2,1 the variable moment of momentum depends on the initial moment of momentum $v^{\prime}{ }_{\varphi} r^{\prime}$, its value at the drain diameter $v_{\varphi_{e}} r_{e}$, and a dimensionless parameter $\mathrm{A}=Q_{e} /(2 \pi . h . \nu)\left(Q_{e}=\right.$ discharge, $h=$ water depth, $v=$ riscosity).

\section{The free water surface}

The first Navier-Stokes equation (eq. 1) can be integrated under the same assumptions as above. Two relations, for the area $r \geq r_{c}$ and $r \leq r_{e}$ were found by which the water surface profile can be determined. For $r \geq r_{e}$ and $A>10$ the following expression can be used:

(where $H=$ specific energy head).

$$
h=\mathrm{H}-\frac{v_{r}^{2}}{2 g}-\frac{g\left(1-e^{-(A / 2)}\right)^{2}}{2 g}
$$

Some of the simplifying assumptions made here do not hold good where $\mathrm{A}<10$. Since small A-values are of minor importance, no further improvements are necessary. For $r \leq r$ the integration is not possible in a closed form. The non-integrable expression is expanded into a series and integrated member by member (eq. 11,1; 11,2 and 11,3$)$. The summation $\Sigma$ of the very slowly converging and alternating series is made graphically. Figure 2 shows characteristic $r / r_{c}$-curves of $\Sigma$ against $A$. As the final solution, the equation for the free surface in the area $r \leqq r_{e}$ is given by :

$$
h=h_{0}-\frac{v_{r}^{2}}{2 g}+\frac{v_{\varphi_{R}}{ }^{2}}{g\left(1-e^{-(\Lambda / 2)}\right)^{2}} \Sigma
$$

with the ordinate $h_{0}$ at the axis of the drain.

\footnotetext{
* Chief Engineer at Theodor Rehbock-Flusslaboratorium der Technischen Hochschule Karlsruhe.
} 


\section{G. HOLTORFF}

One can state that in the limiting cases $\mathrm{A}=0$ and $\mathrm{A}=\infty$ the theoretical results become identical with the valid laws. In order to check both equations 1,4 for finite A-values the results were tested by experimental curves and were found to agree fairly well.

\section{The conditions of similarify.}

From the parameters determining the shape of the free surface the following system of dimensionless combinations can be deduced :

$$
\begin{aligned}
& \text { for } r \geqq r_{e} \quad \frac{\mathrm{H}-h}{r_{e}}=f\left(\frac{r^{\prime}}{r_{e}} ; \mathscr{F}_{\text {rot } r^{\prime}}\right) \\
& \text { for } r \leqq r_{e} \quad \frac{h-h_{0}}{r_{e}}=f\left(\frac{r^{\prime}}{r^{\prime}} ; \mathfrak{g}_{\operatorname{rot} r_{e}} ; \mathcal{R}_{\operatorname{rad} r_{e}}\right) \\
& \text { with } \mathscr{G}_{\mathrm{rot} r^{\prime}}=\frac{v_{e}^{\prime}{ }^{2}}{g r^{\prime}}, \mathscr{F}_{\operatorname{rot} r_{c}}=\frac{v_{\varphi e}{ }^{2}}{g \cdot r_{e}} \text { and } \mathcal{A}_{\mathrm{rad} r_{e}}=\frac{v_{r_{e}}-r_{e}}{v}=\mathrm{A}
\end{aligned}
$$

With this relationship, and considering equation 1,4 and some substantiated suppositions, one obtains the equations :

$$
\begin{gathered}
\text { for } r \leqq r_{e} \quad \frac{h-h_{0}}{r_{e}}=\frac{r_{e}^{2}}{2 r^{2}} \mathscr{F}_{\operatorname{rot} r_{e}} \\
\text { for } r \geqq r_{e} \quad \frac{h-h_{0}}{r_{e}}=\mathscr{J}_{\operatorname{rot} r_{o}} \sum\left(\mathcal{A}_{\operatorname{rad} r_{\mathrm{e}}} ; \frac{r}{r_{e}}\right)
\end{gathered}
$$

as both the condition of similarity and equation of the free surface, respectively. The difficulty for experimental hydraulic research results from the existence of two different conditions (for $r \geqq r_{e}$ and $r \leqq r_{e}$ ) for the same experimental task. How to solve this difficulty is different in each case. However, it may be seen from the above equation for $r \leqq r_{e}$ that the radial velocities are essentially greater than is expected according Froude's law. This fact tends to agree with experience of other investigators. 